\title{
Tuberculosis and Human Immune Deficiency Virus Co-infection in Debre Markos Referral Hospital in Northwest Ethiopia: A Five Years Retrospective Study
}

Ahmed Esmael $^{1 *}$, Girma Tsegaye ${ }^{2}$, Moges Wubie ${ }^{1}$ and Mengistu Endris ${ }^{3}$

1Department of Microbiology, Immunology \& Parasitology, Debre Markos University, Ethiopia

${ }^{2}$ Department of public health, Debre Markos University, Ethiopia

${ }^{3}$ Department of Medical Microbiology, University of Gondar, Ethiopia

\section{Abstract}

Background: Tuberculosis (TB) and Human Immunodeficiency Virus (HIV) are major public health problems, especially in Sub-Saharan Africa including Ethiopia. So, updated information on TB-HIV co-infection might be important for the planning, resource allocation, prevention and control activities.

Objective: To determine the TB-HIV co-infection and possible associated factors among patients attended TB clinic in five years (2008-2013) at a referral Hospital in Northwest Ethiopia.

Methods: A retrospective study was conducted among 717 TB patients who are registered at DOTs clinic of Debre Markos referral hospital. Socio-demographic and clinical data of the study participants were collected from the TB log from the DOTS clinic. Data were entered and analyzed using SPSS version 16 software.

Results: The prevalence of TB-HIV co-infection was 44\% (321/717). The trend of co-infection was decreased from 2008/9 $179(49.2 \%)$ to $2012 / 1329(44.6 \%)$. Types of TB and age group were significantly associated with TB-HIV co-infection.

Conclusion: The prevalence of TB-HIV co-infection was high. Therefore, actions targeting (health education and early case detection) on those predictors are necessary to effectively reduce TB-HIV co-infection and strengthen the collaborative activities.

Keywords: Tuberculosis; TB-HIV co-infection; Western ethiopia

\section{Introduction}

According to World Health Organization (WHO), there were an estimated 8.7 million incident cases of TB (range, 8.3 million-9.0 million) globally [1]. Africa, more specifically Sub-Saharan Africa, faces the worst TB epidemic, where TB associated morbidity and mortality occurs mainly in the economically productive age group (15-60 years) [2].

Available data indicated, Ethiopia has been one of the 22 high TB burden countries [3] with an incidence and prevalence rate of 300 and 470 cases per 100,000 populations respectively. Among all new TB cases notified to federal ministry of health, $30 \%$ were smear positive $[4,5]$.

Moreover, the national adult human immunodeficiency virus (HIV) prevalence infection in Ethiopia was 1.5\% [6]. In 2009, the HIV/ AIDS Prevention and Control Office (HAPCO) estimated that there were around $1,116,216$ people living with HIV. There were also a total 855,720 AIDS orphans in the country $[7,8]$.

TB is the leading cause of death among people living with HIV. Almost one in four deaths among people with HIV is due to TB. In 2010, 350,000 people died of HIV-associated TB [9].

TB and HIV co-infection has been well documented in Sub-Saharan African set up [10]. Studies showed that the prevalence of co-infection was $31.25 \%$, even the rate was as high as $80 \%[11,12]$. Studies in Ethiopia also depicted that the co-infection condition has being on increasing rate $6.6 \%$, to $75 \%$ in the previous decade (1990-2000) [13-18].

TB-HIV co-infection has been associated with different age group [17], geographical location [19] and clinical presentation of TB [20-23]. For instance, the high prevalence of HIV was observed in patients with extra-pulmonary and smear negative tuberculosis than smear positive cases $[19,22]$

East Gojam is one of the 11 zones in Amhara region with a total population of $2,153,937$. Only $8 \%$ of the inhabitants of have access to electricity. Majority of the inhabitants were farmer (85\%). The case detection and treatment success rate of TB in the zone was $26 \%$ and $86 \%$, respectively $[24,25]$.

Despite this needs, there is no adequate and recent data in Ethiopia especially in this study area. Assessment of TB and HIV co-infection might be important for the planning, resource allocation, prevention and control activities. Therefore this study aimed to assess TB-HIV coinfection and the possible associated factors among patients attended TB clinic in Debre Markos Referral Hospital, Northwest Ethiopia.

\section{Methods}

\section{Study setting}

The study was conducted at Debre Markos Referral Hospital in

*Corresponding author: Ahmed Esmael, Department of Microbiology, Immunology \& Parasitology, Debre Markos University, Ethiopia, Tel: +251-058-771-6768; E-mail: esmaelahmed8@gmail.com

Received October 18, 2013; Accepted November 18, 2013; Published November 23, 2013

Citation: Esmael A, Tsegaye G, Wubie M, Endris M (2013) Tuberculosis and Human Immune Deficiency Virus Co-infection in Debre Markos Referral Hospital in Northwest Ethiopia: A Five Years Retrospective Study. J AIDS Clin Res 4: 263. doi: 10.4172/2155-6113.1000263

Copyright: @ 2013 Esmael A, et al. This is an open-access article distributed unde the terms of the Creative Commons Attribution License, which permits unrestricted use, distribution, and reproduction in any medium, provided the original author and source are credited. 
Debre Markos, which is $300 \mathrm{~km}$ away from Addis Ababa. The hospital provides health service to more than 3.5 million populations in its catchments.

In the hospital, DOTS clinic is operating under the National Tuberculosis and Leprosy Program (NTLCP) of Ethiopia, under which patients are diagnosed with tuberculosis by examination of morningspot sputum smears by Zeihel-Nieelsen staining, for the presence of Acid fast bacilli (AFB), chest radiographs, and for EPTB, pathological investigations were used. A patient with at least two sputum specimens who were positive for acid-fast bacilli is smear positive pulmonary TB. A patient with symptoms suggestive of $\mathrm{TB}$ and radiographic abnormalities consistent with pulmonary TB is smear negative pulmonary TB. EPTB is confirmed by taking needle aspiration from organ other than $\mathrm{TB}$, run $\mathrm{AFB}$ and radiographic abnormalities.

Patients are referred to the DOTS clinic where they are registered and treated according to the National Tuberculosis and Leprosy Control Program (NTLCP) [8].

\section{Study design and data collection}

We analyzed a five year retrospective records of 717 tuberculosis patients registered at Debre Markos referral Hospital's DOTS clinic from June 2008 to August 2013. Weight of patient was measured by weight balance. The reviewed documents contained basic information such as patient's age, sex, weight address, TB type, and HIV status.

\section{Inclusion and exclusion criteria}

Inclusion criteria: All data from 2008-2013 in DOTS clinic of Debre Markos referral hospital.

Exclusion criteria: Incomplete data on log book of DOTS clinic.

\section{Definitions}

According to the standard definitions of the National Tuberculosis and Leprosy Control Program guideline (NLCP) adopted from WHO [8], the following clinical case definitions were used:

Incomplete data: If any one of the information which was reviewed on log book (patient's age, sex, address, TB type, weight and HIV status) was missed.

\section{Smear-positive pulmonary TB}

A patient with at least two sputum specimens which were positive for acid-fast bacilli (AFB) by microscopy, or a patient with only one sputum specimen which was positive for AFB by microscopy, and chest radiographic abnormalities consistent with active pulmonary TB.

\section{Smear-negative pulmonary TB}

A patient with symptoms suggestive of $\mathrm{TB}$, with at least two sputum specimens which were negative for AFB by microscopy, and with chest radiographic abnormalities consistent with active pulmonary TB (including interstitial or miliary abnormal images), or a patient with two sets of at least two sputum specimens taken at least two weeks apart, and which were negative for AFB by microscopy, and radiographic abnormalities consistent with pulmonary $\mathrm{TB}$ and lack of clinical response to one week of broad spectrum antibiotic therapy.

\section{Extrapulmonary TB}

This included tuberculosis of organs other than the lungs, such as lymph nodes, abdomen, genitourinary tract, skin, joints and bones, meninges, etc. Diagnosis of EPTB was based on fine needle aspiration cytology or biochemical analyses of cerebrospinal/pleural/ascitic fluid or histopathological examination or strong clinical evidence consistent with active extrapulmonary tuberculosis, followed by a decision of a clinician to treat with a full course of anti-tuberculosis chemotherapy. In all the cases of EPTB, sputum examinations and chest radiographs were used to investigate the involvement of lung parenchyma. This hospital lacks the facilities for culture and drug susceptibility testing.

Statistical analysis: After data collection, the data were entered and coded in to Epi data version 3.1 and exported to SPSS version 16.0 statistical software. Data were cleaned for inconsistencies and missing values. Data cleaning was made by doing descriptive statistics like frequency by removing missing/conflicting ideas and responses to questions about relevant information. Recoding and re-categorizing were made for relevant variables. Frequencies and summary statistics (mean, standard deviation, and percentage) were used to describe the study population in relation to socio-demographic and other relevant variables. The degree of association between dependent and independent variables was assessed using adjusted odds ratio with $95 \%$ confidence interval. A bivariate and multivariate logistic regression was performed to assess statistical association between dependent and independent variable. It was done by entering each independent variable separately into bivariate analysis. Then, all variables were entered for the multivariate logistic regression model. In the multivariate logestic regression models significant association was declared at $p$-value less than 0.05 . Finally data was interpreted with the existed data by referring to literature reviews.

Ethical considerations: Ethical clearance was obtained from Research and Publication Directorate Office of Debre Markos University, Ethiopia.

\section{Results}

\section{Characteristics of study participants}

A total of 717 TB patients were enrolled in this study. Of whom, $371(51.7 \%)$ were males with mean age of 30.6 years. The mean initial weight during intensive anti-tuberculosis treatment was $45.2 \mathrm{KGs}$ (SD \pm 22.9 ). Most of the study participants were urban resident 457 (63.7\%), new cases $705(98.3 \%)$ and had smear negative pulmonary TB 433 (60.4\%) (Table 1).

\begin{tabular}{|c|c|c|c|}
\hline Characteristics & & Frequency & Percent \\
\hline \multirow{2}{*}{ Sex } & Male & 371 & 51.7 \\
\hline & Female & 346 & 48.3 \\
\hline \multirow{3}{*}{ Age } & $<18$ & 124 & 17.3 \\
\hline & $18-30$ & 287 & 40 \\
\hline & $\geq 31$ & 306 & 42.7 \\
\hline \multirow{2}{*}{ Residenc } & Urban & 457 & 63.7 \\
\hline & Rural & 259 & 36.3 \\
\hline \multirow{3}{*}{ Category } & New & 705 & 98.3 \\
\hline & Relapse & 11 & 1.5 \\
\hline & Default & 1 & 0.1 \\
\hline \multirow{3}{*}{ TB Type } & Smear positive Pulmonary TB & 103 & 14.4 \\
\hline & Smear negative Pulmonary TB & 433 & 60.4 \\
\hline & Extra-pulmonary TB & 181 & 25.2 \\
\hline Over all & & 717 & 100 \\
\hline
\end{tabular}

Table 1: Characteristics of the study participants registered at DOTS clinic at Debre Markos referral hospital from June 2013 to August 2013. 


\section{TB and HIV co-infection}

The prevalence of TB and HIV co-infection was $44.8 \%$ (321/717) (Table 3). The highest rate of co-infection was observed among smear negative pulmonary TB $32.4 \%$ (206/717), urban residents $28.8 \%$ (206/717) and 18-30 age groups 17\% (122/717) (Table 2). The trend of TB-HIV co-infection was decreased from 2008 to 2012 while raise in 2013 (Figure 1 and Table 2).

\section{Associated factors of TB-HIV co-infection}

In multivariate analysis extra pulmonary TB and age group (18-30, 31-40 and 41-50) independently associated with TB -HIV co-infection $(\mathrm{P}<0.05)$ (Table 2).

\section{Discussions}

Currently, the number of TB cases has been increased by several folds especially in sub-Saharan African set up. The presence of high HIV infection is claimed as the main risk factor for the increment of TB patients [3,9].

\section{TB-HIV}

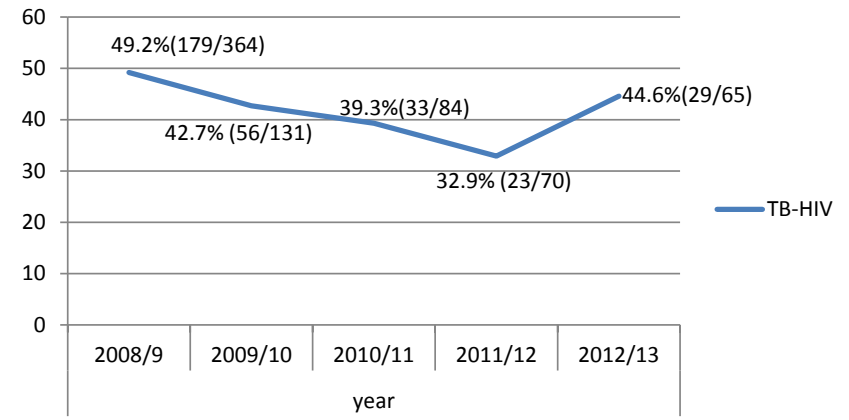

Figure 1: Trend of TB and TB-HIV co-infection at Debre Markos referral hospital from June 2013 to August 2013.
In the present, study, the prevalence of TB and HIV co-infection was $44.8 \%$. In line with these studies in African countries showed that the co-occurrence of both infections is well established fact. The prevalence of co-infection also depends on the prevalence of HIV in a given country [11-18]. For instance, study in Cameron [26], in Ghana [27] and in Zimbabwe [28] depicted that the prevalence of cooccurrence was $51.6 \%, 46.2 \%$, and $44 \%$, respectively.

In the present study, majority of study participants who showed TB/HIV co-infection was pulmonary negative $232(72.3 \%)$ and extra pulmonary type 51 (15.9\%). The occurrence of TB/HIV co-infection was 2.4 times higher in extra pulmonary tuberculosis cases than pulmonary positive cases. This finding was consistent with Daniel et al. in Ethiopia and Sharma et al. in India which showed that high prevalence of coinfection was observed in patients with extra-pulmonary and smear negative tuberculosis than smear positive cases $[19,21]$.

Studies, especially in sub-Saharan African countries showed that the number of HIV infected TB cases were higher in urban than rural areas [19,26-28]. Possibly this might be due to a presence of high prevalence of HIV infection [8,9]. However in our findings, there was no significance difference in the rate of TB/HIV co-infection.

In the present study age group (18-30 and $\geq 31)$ had statistical significance association with the occurrence of TB/HIV co-infection. This finding was in agreement with studies conducted by in Ethiopia [17], in Cameron [26], and in Ghana [27].

Currently there has been a conflicting report of occurrence of TB/ HIV co-infection with regards to sex. For instance Adji et al. in Cameron showed that women had significantly more TB/HIV co-infected than men (42\%) [26]. However Demissie et al. in Ethiopia indicated that TB/ HIV co-infection did not have any significance difference with sex [17]. Our study also depicted similar findings.

Moreover, studies in Ethiopia indicated that the prevalence of TB/ HIV co-infection was $6.6 \%$ in $1990,20-25 \%$ in $1995,44.4 \%$ in 1996 ,

\begin{tabular}{|c|c|c|c|c|c|c|}
\hline \multirow{2}{*}{ Variables } & \multicolumn{2}{|c|}{ TB-HIV co-infection } & \multirow{2}{*}{ COR $(95 \% \mathrm{Cl})$} & \multirow{2}{*}{ P-value } & \multirow{2}{*}{ AOR $(95 \% \mathrm{Cl})$} & \multirow{2}{*}{ P-value } \\
\hline & Yes & No & & & & \\
\hline \multicolumn{7}{|l|}{ Sex } \\
\hline Male & 161 & 210 & 1 & ---- & 1 & --- \\
\hline Female & 160 & 186 & $0.89(0.6-1.20)$ & 0.46 & $0.78(0.54-1.06)$ & 0.12 \\
\hline \multicolumn{7}{|l|}{ Age (years) } \\
\hline$<18$ & 35 & 89 & 1 & --- & 1 & ---- \\
\hline $18-30$ & 122 & 165 & $2.93(1.8-4.61)$ & 0.007 & $2.52(1.25-3.14)$ & 0.005 \\
\hline$\geq 31$ & 164 & 142 & $1.56(1.1-2.16)$ & 0.000 & $1.34(1.1-2.05)$ & 0.001 \\
\hline \multicolumn{7}{|l|}{ Residence } \\
\hline Urban & 206 & 251 & $0.96(0.7-1.31)$ & 0.81 & $0.71(0.63-1.28)$ & 0.46 \\
\hline Rural & 115 & 145 & 1 & ---- & 1 & ---- \\
\hline \multicolumn{7}{|l|}{ Type of TB } \\
\hline Smear positive PTB & 38 & 65 & 1 & ---- & 1 & --- \\
\hline Smear negative PTB & 232 & 201 & $0.50(0.3-0.79)$ & 0.02 & $0.74(0.35-1.77)$ & 0.59 \\
\hline Extra PTB & 51 & 130 & $1.49(0.8-2.49)$ & 0.13 & $2.44(1.01-6.14)$ & 0.03 \\
\hline
\end{tabular}

Table 2: Bi and Multivariate analysis of TB/HIV co-infection $(n=717)$ with different variables at Debre Markos referral hospital from June 2013 to August 2013.

\begin{tabular}{|l|c|c|c|c|c|}
\hline \multirow{2}{*}{ TB Cases } & \multicolumn{3}{|c|}{ Year } & \multicolumn{2}{|c|}{ Total } \\
\cline { 2 - 5 } & $\mathbf{2 0 0 8 / 9}$ & $\mathbf{2 0 0 9 / 1 0}$ & $\mathbf{2 0 1 0 / 1 1}$ & $\mathbf{2 0 1 1 / 1 2}$ & $\mathbf{2 0 1 2 / 1 3}$ \\
\hline TB-HIV co-infection & $179(49.2)$ & $56(42.7)$ & $33(39.3)$ & $23(32.9)$ & $323(44.8)$ \\
\hline HIV negative TB cases & $185(50.8)$ & $75(57.3)$ & $51(60.7)$ & $47(67.1)$ \\
\hline Total & $\mathbf{3 6 4 ( 5 1 . 0 )}$ & $\mathbf{1 3 1 ( 1 8 . 3 )}$ & $\mathbf{8 4 ( 1 1 . 8 )}$ & $\mathbf{7 0 ( 9 . 8 )}$ & $39(55.4)$ \\
\hline
\end{tabular}

Table 3: Trend of TB and TB-HIV co-infection at Debre Markos referral hospital from June 2008 to August 2013. 
45.3 in 1997 and $52.1 \%$ in 2007 [13-18]. However in our study, the co-infection rate was continuously decreased from 2009 to 2012 while somewhat rise during 2013. The possible explanation for this might be due to the current strategic plan of the federal minister of health of Ethiopia, which expands health facilities across the country. As a result the number of cases who notified in the hospital might be reduced.

\section{Conclusion and Recommendation}

The prevalence of TB and HIV co-infection was higher than most previous studies in Ethiopia. The rate of TB-HIV co-infection was significantly associated with type of TB and age groups (18-30, $\geq 31$ ) in our study. Therefore, actions targeting (health education and early case detection) on those predictors are necessary to effectively reduce TB-HIV co-infection.

\section{Competing Interests}

This work was ethically cleared by RPO of Debre Markos University. There is no any competing of interest. No future financial aid was received from any organization for publication or other interest.

\section{Author's Contributions}

$\mathrm{AE}$ and ME: conception and initiation of the study, design, implementation, analysis and writing. GT and MW: implementation, and analysis. All authors read and approved the final manuscript.

\section{Acknowledgements}

Our acknowledgements go to A to Tariku Belachew, director of Debre Markos hospital and all members of RPO of Debre Markos University that facilitating all the bureaucratic procedures smoothly and swiftly. The authors also thank data collectors, Debre Markos hospital laboratory and DOTs clinic staffs for their unreserved support during the study period.

\section{References}

1. WHO (2012) Gains in TB control at risk due to 3 million missed patients and drug resistance. Global tuberculosis report.

2. WHO (2000) Regional Office for South-East Asia, New Delhi. TB in South-East.

3. Prasad R (2010) Multidrug and extensively drug-resistant TB (M/XDR-TB): problems and solutions. Indian J Tuberc 57: 180-191.

4. WHO (2010) Tuberculosis country profiles.

5. Ministry of Health (2008) National tuberculosis control program in Ethiopia.

6. Demissie BS, Solomon AW (2011) Magnitude and causes of childhood blindness and severe visual impairment in Sekoru District, Southwest Ethiopia: a survey using the key informant method. Trans R Soc Trop Med Hyg 105: 507-511.

7. Federal Ministry of Health $(\mathrm{FMoH})$ : National Consensus Meeting on Single Point HIV Prevalence Estimate. Palace Hotel, Adama, Ethiopia.

8. Ministry of Health (2010/11-2014/15) Federal Democratic Republic of Ethiopia.

9. WHO (2011/2012) Tuberculosis Global Facts.

10. World Health organization (2004) The world health report 2004 - changing history.

11. WHO report (2010) Global tuberculosis control

12. Gao J, Zheng P, Fu H (2013) Prevalence of TB/HIV co-infection in countries except China: a systematic review and meta-analysis. PLoS One 8: e64915.
13. Gellete A, Kebede D, Berhane Y (1997) Tuberculosis and HIV infection in Southern Ethiopia. Ethiop J Health Dev 11: 51-59.

14. Kefene H, Zewdie D, Desta B, Kebede T (1990) The prevalence of HIVAntibodies in 106 TB patients. Ethiop J Health Dev 4: 197-200.

15. Mitike G, Kebede D, Yeneneh H (1997) HIV infection and anti-tuberculosis drug resistance among pulmonary tuberculosis patients in Harrar Tuberculosis Centre, Ethiopia. East Afr Med J 74: 154-157.

16. Madebo T, Nysaeter G, Lindtjørn B (1997) HIV infection and malnutrition change the clinical and radiological features of pulmonary tuberculosis. Scand J Infect Dis 29: 355-359.

17. Demissie M, Lindtjørn B, Tegbaru B (2000) Human immunodeficiency virus (HIV) infection in tuberculosis patients in Addis Ababa. Ethiop. J. Health Dev 14: 277-282.

18. Kassu A, Mengistu G, Ayele B, Diro E, Mekonnen F, et al. (2007) Coinfection and clinical manifestations of tuberculosis in human immunodeficiency virusinfected and uninfected adults at a teaching hospital, northwest Ethiopia. J Microbiol Immunol Infect 40: 116-122.

19. Datiko DG, Yassin MA, Chekol LT, Kabeto LE, Lindtjørn B (2008) The rate of TB-HIV co-infection depends on the prevalence of HIV infection in a community. BMC Public Health 8: 266.

20. Sume GE, Etogo D, Kabore S, Gnigninanjouena O, Epome SS, et al. (2008) Sero prevalence of human immunodeficiency virus infection among tuberculosis patients in the Nylon district hospital tuberculosis treatment centre. East Afr Med J 85: 529-536.

21. Sharma SK, Mohan A (2004) Extrapulmonary tuberculosis. Indian J Med Res 120: 316-353.

22. Golden MP, Vikram HR (2005) Extrapulmonary tuberculosis: an overview. Am Fam Physician 72: 1761-1768.

23. Gebremariam MK, Bjune GA, Frich JC (2011) Lay beliefs of TB and TB/HIV coinfection in Addis Ababa, Ethiopia: a qualitative study. BMC Res Notes 4: 277.

24. Central Statistical Agency of Ethiopia (CSA).

25. Amhara National Regional State Health Bureau Fifth National TB Research Conference.

26. Pefura Yone EW, Kuaban C, Kengne AP (2012) HIV testing, HIV status and outcomes of treatment for tuberculosis in a major diagnosis and treatment centre in Yaounde, Cameroon: a retrospective cohort study. BMC Infect Dis 12: 190.

27. Adjei AA, Adiku TK, Ayeh-Kumi PF, Hesse IF (2006) Prevalence of human immunodeficiency virus infection among tuberculosis suspect patients in Accra Ghana. West Afr J Med 25: 38-41.

28. Houston S, Ray S, Mahari M, Neill P, Legg W, et al. (1994) The association of tuberculosis and HIV infection in Harare, Zimbabwe. Tuber Lung Dis 75 : 220-226. 